PROCEEDINGS OF THE

AMERICAN MATHEMATICAL SOCIETY

Volume 136, Number 6, June 2008, Pages 2031-2041

S 0002-9939(08)09280-0

Article electronically published on February 11, 2008

\title{
EQUIVARIANT HOPF BIFURCATION FOR NEUTRAL FUNCTIONAL DIFFERENTIAL EQUATIONS
}

\author{
SHANGJIANG GUO AND JEROEN S. W. LAMB \\ (Communicated by Carmen C. Chicone)
}

\begin{abstract}
In this paper we employ an equivariant Lyapunov-Schmidt procedure to give a clearer understanding of the one-to-one correspondence of the periodic solutions of a system of neutral functional differential equations with the zeros of the reduced bifurcation map, and then set up equivariant Hopf bifurcation theory. In the process we derive criteria for the existence and direction of branches of bifurcating periodic solutions in terms of the original system, avoiding the process of center manifold reduction.
\end{abstract}

\section{INTRODUCTION}

Suppose that $\tau \geq 0$ is a given constant (or possibly infinity), and $\mathbb{R}^{n}$ is the $n$ dimensional Euclidean space with norm $|\cdot|$. Let $C\left([-\tau, 0], \mathbb{R}^{n}\right)$ denote the Banach space of continuous mappings from $[-\tau, 0]$ into $\mathbb{R}^{n}$ equipped with the supremum norm $\|\phi\|=\sup _{-\tau \leq \theta \leq 0}|\phi(\theta)|$ for $\phi \in C\left([-\tau, 0], \mathbb{R}^{n}\right)$. If $a \geq 0$, and $x:[-\tau, a] \rightarrow \mathbb{R}^{n}$ is a continuous mapping, then for any $t \in[0, a], x_{t} \in C\left([-\tau, 0], \mathbb{R}^{n}\right)$ is defined by $x_{t}(\theta)=x(t+\theta)$ for $\theta \in[-\tau, 0]$. In this paper, we consider the following parameterized system of neutral functional differential equations (NFDEs)

$$
\frac{d}{d t} h\left(\alpha, x_{t}\right)=f\left(\alpha, x_{t}\right)
$$

where $h, f: \mathbb{R} \times C\left([-\tau, 0], \mathbb{R}^{n}\right) \rightarrow \mathbb{R}^{n}$ are two continuously differentiable mappings satisfying that $f(\alpha, 0)=0$ for all $\alpha \in \mathbb{R}$. We say that (1.1) is equivariant with respect to a group $\Gamma$ if there exists a representation $\varrho$ of $\Gamma$ such that

$$
h(\alpha, \varrho(\gamma) \phi)=\varrho(\gamma) h(\alpha, \phi), \quad f(\alpha, \varrho(\gamma) \phi)=\varrho(\gamma) f(\alpha, \phi)
$$

for $(\alpha, \gamma, \phi) \in \mathbb{R} \times \Gamma \times C\left([-\tau, 0] ; \mathbb{R}^{n}\right)$, where $\varrho(\gamma) \phi \in C\left([-\tau, 0] ; \mathbb{R}^{n}\right)$ is given by $(\varrho(\gamma) \phi)(s)=\varrho(\gamma) \phi(s)$ for $s \in[-\tau, 0]$. Recall that a representation $\varrho$ of a group $\Gamma$ is a group homomorphism $\varrho: \Gamma \rightarrow G L(n, \mathbb{R})$. Condition (1.2) implies that

Received by the editors January 9, 2007

2000 Mathematics Subject Classification. Primary 34K18; Secondary 34K20.

Key words and phrases. Lyapunov-Schmidt reduction, equivariant Hopf bifurcation, neutral functional differential equations, Lie group.

The first author was supported in part by a China postdoctoral fellowship of the UK Royal Society, by the NNSF of China (Grant No. 10601016), by the program for New Century Excellent Talents in University of the Education Ministry of China (Grant No. [2007]70), and by the NSF of Hunan (Grant No. 06JJ3001).

The second author was supported in part by the UK Engineering and Physical Sciences Research Council (EPSRC).

(C)2008 American Mathematical Society Reverts to public domain 28 years from publication 
system (1.1) is invariant under the transformation $(x, t) \rightarrow(\varrho(\gamma), t)$. Namely, $x(t)$ is a solution of (1.1) if and only if $\varrho(\gamma) x(t)$ is a solution. Throughout this paper we always assume that $\Gamma$ is a compact Lie group and that system (1.1) is $\Gamma$-equivariant.

The phenomenon of Hopf bifurcation concerns the birth of a periodic solution from an equilibrium solution through a local oscillatory instability. The existence of such a bifurcation was found in the context of ordinary differential equations (ODEs) by Hopf [1] in 1942, after whom this type of bifurcation has been subsequently named. For delay differential equations (DDEs), the first results on Hopf bifurcation date back to the work by Chafee [1 in 1971. However, according to Hale 9], the first proof of the Hopf bifurcation theorem for DDEs under analytically computable conditions was presented by Chow and Mallet-Paret in 1977 [2]. The first results on equivariant Hopf bifurcation of DDEs date back to the work by $\mathrm{Wu}$ [14. In [12, Krawcewicz, $\mathrm{Ma}$ and $\mathrm{Wu}$ employ equivariant degree theory developed by Geba et al. (cf. [4) to study the existence, multiplicity and global continuations of symmetric periodic solutions for a one-parameter family of NFDEs with dihedral symmetry.

In the standard Hopf bifurcation theory $(9,10,13])$, the central hypothesis is that at the critical value of the bifurcation parameter the infinitesimal generator has a complex conjugate pair of simple purely imaginary eigenvalues. The presence of symmetry may cause purely imaginary eigenvalues to arise with higher multiplicities, which causes the bifurcation problem to become more complicated; see for instance [6]. The most common approach to study bifurcation problems in functional differential equations involves the computation of (normal forms of) reduced bifurcation equations on center manifolds. For example, Faria and Magalhães [3], and Weedermann [13] compute the reduced system on the center manifold associated with the pair of purely imaginary solutions of the characteristic equation.

In this paper, we present a treatment of generic codimension-one Hopf bifurcation for equivariant NFDEs on the basis of equivariant Lyapunov-Schmidt reduction, following the spirit of the treatment of Golubitsky and Stewart [5, 6] in the case of equivariant ODEs. Moreover, our results generalize those obtained by Wu 14 and Krawcewicz, Ma and $\mathrm{Wu}[12$. In the process we obtain explicit expressions in terms of the original system that determine the monotonicity of the period and Hopf bifurcation direction of branches of bifurcating symmetric periodic solutions. With these expressions at our disposal, the study of equivariant Hopf bifurcation in explicit examples can be performed without having to resort to lengthy computations associated to center manifold reduction.

\section{MAIN RESUlts}

Let $D(\alpha), L(\alpha): C\left([-\tau, 0] ; \mathbb{R}^{n}\right) \rightarrow \mathbb{R}^{n}$ be the two linearized operators of $h(\alpha, \cdot)$ and $f(\alpha, \cdot)$, respectively. Furthermore, we assume that $D(\alpha)$ is atomic at zero (see [9] for more details). By the Riesz representation theorem, there exists an $n \times n$ matrix-valued function $\mu, \eta:[-\tau, 0] \rightarrow \mathbb{R}^{n^{2}}$ whose components each have bounded variation and such that for $\varphi \in C\left([-\tau, 0], \mathbb{R}^{n}\right)$,

$$
D(\alpha) \varphi=\varphi(0)-\int_{-\tau}^{0} d \mu(\alpha, \theta) \varphi(\theta), \quad L(\alpha) \varphi=\int_{-\tau}^{0} d \eta(\alpha, \theta) \varphi(\theta)
$$


where $\operatorname{Var}_{[s, 0]} \mu(\alpha, \theta) \rightarrow 0$ as $s \rightarrow 0$ (see 9 for more details). According to Hale 9], for each fixed $\alpha$, the linear system

$$
\frac{d}{d t} D(\alpha) x_{t}=L(\alpha) x_{t}
$$

generates a strongly continuous semigroup of linear operators with infinitesimal generator $\mathcal{A}_{\alpha}$. The spectrum of $\mathcal{A}_{\alpha}$, denoted by $\sigma\left(\mathcal{A}_{\alpha}\right)$, is the point spectrum. Moreover, $\lambda$ is an eigenvalue of $\mathcal{A}_{\alpha}$, i.e., $\lambda \in \sigma\left(\mathcal{A}_{\alpha}\right)$, if and only if $\lambda$ satisfies that $\operatorname{det} \Delta(\alpha, \lambda)=0$, where the characteristic matrix $\Delta(\tau, \lambda)$ is given by

$$
\Delta(\alpha, \lambda)=\lambda D(\alpha)\left(e^{\lambda(\cdot)} \mathrm{Id}\right)-L(\alpha)\left(e^{\lambda(\cdot)} \mathrm{Id}\right) .
$$

It is well known that $\phi \in C\left([-\tau, 0] ; \mathbb{C}^{n}\right)$ is an eigenvector of $\mathcal{A}_{\alpha}$ associated with the eigenvalue $\lambda$ if and only if $\phi(\theta)=e^{\lambda \theta} b$ for $\theta \in[-\tau, 0]$ and some vector $b \in \mathbb{R}^{n}$ such that $\Delta(\alpha, \lambda) b=0$. Let $E_{\alpha, \lambda}$ be the eigenspace of $\mathcal{A}_{\alpha}$ associated with the eigenvalues $\lambda$ and $\bar{\lambda}$. Assume that $\mathcal{A}_{0}$ has a pair of purely imaginary eigenvalues $\pm i \beta_{0}$. The symmetry group $\Gamma$ often causes purely imaginary eigenvalues to be multiple. So, we always assume that

(H1) $\mathcal{A}_{0}$ has a pair of purely imaginary eigenvalues $\pm i \beta_{0}$, each of multiplicity $m$, and all other eigenvalues of $\mathcal{A}_{0}$ are not an integer multiple of $i \beta_{0}$.

In studying the bifurcation problem we wish to consider how the eigenvalues of $\mathcal{A}_{\alpha}$ cross the imaginary axis at $\alpha=0$ and to describe the structure of the associated eigenspace $E_{\alpha, \lambda}$. We consider the following nontrivial restrictions on the corresponding imaginary eigenspace of $\mathcal{A}_{0}$.

(H2) The imaginary eigenspace $E_{0, i \beta_{0}}$ of $\mathcal{A}_{0}$ is $\Gamma$-simple.

Thus, we make use of the implicit function theorem and Lemma 1.5 on p. 265 of [6] and obtain the following results on the multiplicity of this eigenvalue and its associated eigenvectors of $\mathcal{A}_{\alpha}$.

Theorem 2.1. Under conditions (H1)-(H2), for sufficiently small $\alpha$, the infinitesimal generator $\mathcal{A}_{\alpha}$ has one pair of complex conjugate eigenvalues $\sigma(\alpha) \pm i \rho(\alpha)$, each of multiplicity $m$. Moreover, $\sigma$ and $\rho$ are smooth functions of $\alpha$ and satisfy that $\sigma(0)=0$ and $\rho(0)=\beta_{0}$.

In view of (H1), the purely imaginary eigenvalues of $\mathcal{A}_{0}$ have high multiplicity, so the standard Hopf bifurcation theorem cannot be applied directly. So, we first develop the equivariant Lyapunov-Schmidt reduction for (1.1) to consider the existence of periodic solutions. Let $\omega_{0}=2 \pi / \beta_{0}$, and $\mathcal{C}_{\omega_{0}}$ (respectively, $\mathcal{C}_{\omega_{0}}^{1}$ ) be the set of continuous (respectively, differentiable) $n$-dimensional $\omega_{0}$-periodic mappings. If we denote

$$
\|x\|_{0}=\max _{1 \leq i \leq n} \max _{t \in\left[0, \omega_{0}\right]}\left\{\left|x_{i}(t)\right|\right\}
$$

for $x=\left(x_{1}, x_{2}, \ldots, x_{n}\right)^{T} \in \mathcal{C}_{\omega_{0}}$, and $\|x\|_{1}=\max \left\{\|x\|_{0},\|\dot{x}\|_{0}\right\}$ for $x \in \mathcal{C}_{\omega_{0}}^{1}$, then $\mathcal{C}_{\omega_{0}}$ and $\mathcal{C}_{\omega_{0}}^{1}$ are Banach spaces when they are endowed with the norms $\|\cdot\|_{0}$ and $\|\cdot\|_{1}$, respectively. It is easy to see that $\mathcal{C}_{\omega_{0}}$ is a Banach representation of the group $\Gamma \times S^{1}$ with the action given by

$$
(\gamma, \theta) u(t)=\varrho(\gamma) u(t+\theta), \quad \text { for } \quad(\gamma, \theta) \in \Gamma \times S^{1} .
$$


In view of the complexity in analyzing NFDEs, we introduce two kinds of bilinear forms. One is the inner product $\langle\cdot, \cdot\rangle: \mathcal{C}_{\omega_{0}} \times \mathcal{C}_{\omega_{0}} \rightarrow \mathbb{R}$ defined by $\langle v, u\rangle=$ $\frac{1}{\omega_{0}} \int_{0}^{\omega_{0}} \bar{v}^{T}(t) u(t) d t$, for $u, v \in \mathcal{C}_{\omega_{0}}$. The other is $(\cdot, \cdot): C\left([-\tau, 0] ; \mathbb{R}^{n}\right) \times C\left([0, \tau] ; \mathbb{R}^{n}\right) \rightarrow$ $\mathbb{R}$ defined by

$$
\begin{gathered}
(\psi, \varphi)=\bar{\psi}^{T}(0) \varphi(0)-\int_{-\tau}^{0}\left[\frac{d}{d s} \int_{0}^{s} \bar{\psi}^{T}(\xi-s) d \mu(0, \theta) \varphi(\xi) d \xi\right]_{s=\theta} \\
-\int_{-\tau}^{0} \int_{0}^{\theta} \bar{\psi}^{T}(\xi-\theta) d \eta(0, \theta) \varphi(\xi) d \xi
\end{gathered}
$$

for $\psi \in C\left([0, \tau] ; \mathbb{R}^{n}\right)$ and $\varphi \in C\left([-\tau, 0] ; \mathbb{R}^{n}\right)$. Let $\beta \in(-1,1), x(t)=u((1+\beta) t)$. Then equation (1.1) can be rewritten as

$$
(1+\beta) \frac{d}{d t} h\left(\alpha, u_{t, \beta}\right)=f\left(\alpha, u_{t, \beta}\right),
$$

where $u_{t, \beta}(\theta)=u(t+(1+\beta) \theta)$ for $\theta \in[-\tau, 0]$. Define $F: \mathcal{C}_{\omega_{0}}^{1} \times \mathbb{R}^{2} \rightarrow \mathcal{C}_{\omega_{0}}$ by

$$
F(u, \alpha, \beta)=-(1+\beta) \frac{d}{d t} h\left(\alpha, u_{t, \beta}\right)+f\left(\alpha, u_{t, \beta}\right) .
$$

By varying the newly introduced small variable $\beta$, one keeps track not only of solutions of (1.1) with period $\omega_{0}$ but also of solutions with nearby period. In fact, solutions to $F(u, \alpha, \beta)=0$ correspond to $\frac{\omega_{0}}{1+\beta}$-periodic solutions of (1.1). It follows from the $\Gamma$-equivariance of $L$ and $f$ that $F$ is $\Gamma \times S^{1}$-eqivariant:

$$
(\gamma, \theta) F(u, \alpha, \beta)=F((\gamma, \theta) u, \alpha, \beta),
$$

for all $(\gamma, \theta) \in \Gamma \times S^{1}$. Define

$$
\mathcal{L} u=-\frac{d}{d t} D(0) u_{t}+L(0) u_{t}
$$

The elements of $\operatorname{Ker} \mathcal{L}$ correspond to solutions of the linear system $\mathcal{L} u=0$ satisfying $u(t)=u\left(t+\omega_{0}\right)$. For convenience in computation we shall allow functions with range $\mathbb{C}^{n}$ instead of $\mathbb{R}^{n}$. With respect to the inner product $\langle\cdot, \cdot\rangle: \mathcal{C}_{\omega_{0}} \times \mathcal{C}_{\omega_{0}} \rightarrow \mathbb{R}$, the adjoint operator of $\mathcal{L}$ is

$$
\mathcal{L}^{*} u=\frac{d}{d t}\left[u(t)-\int_{-\tau}^{0} d \mu^{T}(0, \theta) u(t-\theta)\right]+\int_{-\tau}^{0} d \eta^{T}(0, \theta) u(t-\theta) .
$$

It follows from $(\mathrm{H} 1)$ that $\operatorname{Ker} \mathcal{L} \cong E_{0, i \beta_{0}}$ and $\operatorname{Ker} \mathcal{L}^{*} \cong E_{0, i \beta_{0}}^{*}$, both of which are $2 m$-dimensional. Here and in the sequel, $\mathcal{L}^{*}$ denotes the adjoint map with respect to the inner product $\langle\cdot, \cdot\rangle$. Furthermore, we have

Lemma 2.2. The spaces $\operatorname{Ker} \mathcal{L}$, Range $\mathcal{L}$, and $\mathfrak{W}=\left(\operatorname{Ker} \mathcal{L}^{*}\right)^{\perp} \cap \mathcal{C}_{\omega_{0}}^{1}$ are $\Gamma \times S^{1}$ invariant subspaces of $\mathcal{C}_{\omega_{0}}$. Moreover, $\mathcal{C}_{\omega_{0}}=\operatorname{Ker} \mathcal{L} \oplus \operatorname{Range} \mathcal{L}$ and $\mathcal{C}_{\omega_{0}}^{\omega_{0}}=\operatorname{Ker} \mathcal{L} \oplus \mathfrak{W}$.

Let $P$ and $I-P$ denote the projection operators defined by

$$
P: \mathcal{C}_{\omega_{0}} \rightarrow \text { Range } \mathcal{L}, \quad I-P: \mathcal{C}_{\omega_{0}} \rightarrow \operatorname{Ker} \mathcal{L} .
$$

Obviously, $P$ and $I-P$ are $\Gamma \times S^{1}$-equivariant. Thus, $F(u, \alpha, \beta)=0$ is equivalent to the following system:

$$
P F(u, \alpha, \beta)=0, \quad(I-P) F(u, \alpha, \beta)=0 .
$$

According to the above direct sum decomposition, for each $u \in \mathcal{C}_{\omega_{0}}^{1}$, there is a unique decomposition such that $u=v+w$, where $v \in \operatorname{Ker} \mathcal{L}$ and $w \in \mathfrak{W}$. Thus, the first equation of (2.4) can be rewritten as

$$
G(v, w, \alpha, \beta) \equiv P F(v+w, \alpha, \beta)=0 .
$$


Notice that $G(0,0,0,0)=P F(0,0,0)=0$ and $D_{w} G(0,0,0,0)=P \mathcal{L}=\mathcal{L}$. When $\mathcal{L}$ is restricted in $\mathfrak{W}$, it is invertible, and so $D_{w} G(0,0,0,0)$ is invertible. Applying the implicit function theorem, we obtain a continuously differentiable $\Gamma \times S^{1}$-equivariant map $W: \operatorname{Ker} \mathcal{L} \times \mathbb{R}^{2} \rightarrow \mathfrak{W}$ such that $W(0,0,0)=0$ and

$$
P F(v+W(v, \alpha, \beta), \alpha, \beta) \equiv 0 .
$$

Substituting $w=W(v, \alpha, \beta)$ into the second equation of (2.4), we have

$$
\mathcal{B}(v, \alpha, \beta) \equiv(I-P) F(v+W(v, \alpha, \beta), \alpha, \beta)=0 .
$$

Thus, we reduce our Hopf bifurcation problem to the problem of finding zeros of the map $\mathcal{B}: \operatorname{Ker} \mathcal{L} \times \mathbb{R}^{2} \rightarrow \operatorname{Ker} \mathcal{L}$. We refer to $\mathcal{B}$ as the bifurcation map of the system (1.1). It follows from the $\Gamma \times S^{1}$-equivariance of $F$ and $W$ that the bifurcation map $\mathcal{B}$ is also $\Gamma \times S^{1}$-equivariant. Moreover, $\mathcal{B}(0,0,0)=0$ and $\mathcal{B}_{v}(0,0,0)=0$.

Finding periodic solutions to (1.1) rests on prescribing in advance the symmetry of the solution we seek. This can often be used to select a subspace on which the eigenvalues are simple. In addition, we should take temporal phase-shift symmetries in terms of the circle group $S^{1}$ into account as well as spatial symmetries. Here, we place emphasis on two-dimensional fixed-point subspaces and assume that

(H3) $\operatorname{dim} \operatorname{Fix}\left(\Sigma, E_{0, i \beta}\right)=2$ for some subgroup $\Sigma$ of $\Gamma \times S^{1}$, (H4) $\sigma^{\prime}(0) \neq 0$.

Assumption (H4) is the transversality condition analogous to those of the standard Hopf bifurcation theorem. Now, we can present our main results about equivariant Hopf bifurcation.

Theorem 2.3. Under conditions (H1)-(H4), in every neighborhood of $(x=0, \alpha=$ 0) system (1.1) has a bifurcation of periodic solutions whose spatio-temporal symmetry can be completely characterized by $\Sigma$.

Proof. We consider the restriction mapping $\widetilde{\mathcal{B}}: \operatorname{Fix}(\Sigma, \operatorname{Ker} \mathcal{L}) \times \mathbb{R}^{2} \rightarrow \operatorname{Ker} \mathcal{L}$ of $\mathcal{B}: \operatorname{Ker} \mathcal{L} \times \mathbb{R}^{2} \rightarrow \operatorname{Ker} \mathcal{L}$ on $\operatorname{Fix}(\Sigma, \operatorname{Ker} \mathcal{L}) \times \mathbb{R}^{2} ;$ i.e.

$$
\widetilde{\mathcal{B}}(v, \alpha, \beta)=(I-P) F(v+W(v, \alpha, \beta), \alpha, \beta)
$$

for $v \in \operatorname{Fix}(\Sigma, \operatorname{Ker} \mathcal{L}), \alpha \in \mathbb{R}$, and $\beta \in \mathbb{R}$. Clearly, $\widetilde{\mathcal{B}}$ is also $\Gamma \times S^{1}$-equivariant and satisfies that

$$
\widetilde{\mathcal{B}}(0,0,0)=0, \quad \widetilde{\mathcal{B}}_{v}(0,0,0)=0 .
$$

Moreover, it is easy to see that $\operatorname{Range} \widetilde{\mathcal{B}} \subseteq \operatorname{Fix}(\Sigma, \operatorname{Ker} \mathcal{L})$. Namely, $\widetilde{\mathcal{B}} \operatorname{maps} \operatorname{Fix}(\Sigma$, $\operatorname{Ker} \mathcal{L}) \times \mathbb{R}^{2}$ to $\operatorname{Fix}(\Sigma, \operatorname{Ker} \mathcal{L})$. Therefore, we only need to consider the existence of nontrivial zeroes of $\widetilde{\mathcal{B}}$.

Without loss of generality, assume that $\operatorname{Fix}(\Sigma, \operatorname{Ker} \mathcal{L})=\operatorname{span}\{q, \bar{q}\}$, where $q(\theta)=A e^{i \beta_{0} \theta}$ and $A \in \mathbb{C}^{n}$ satisfies $\Delta(0, i \beta) A=0$. Obviously, $\operatorname{Fix}(\Sigma, \operatorname{Ker} \mathcal{L})^{*}=$ $\operatorname{Fix}\left(\Sigma, \operatorname{Ker} \mathcal{L}^{*}\right)$. Thus, there exists $B \in \mathbb{C}^{n}$ such that $\bar{B}^{T} A=1$ and $\operatorname{Fix}\left(\Sigma, \operatorname{Ker} \mathcal{L}^{*}\right)=$ $\operatorname{span}\{p, \bar{p}\}$, where $p(\theta)=B e^{i \beta_{0} \theta}$. As stated in Theorem 2.1 for sufficiently small $\alpha$; the infinitesimal generator $\mathcal{A}_{\alpha}$ has one pair of complex conjugate eigenvalues $\lambda(\alpha)=\sigma(\alpha) \pm i \rho(\alpha)$, each of multiplicity $m$. Moreover, there exists a $C^{1}$-continuous function $\widetilde{A}(\alpha)$ such that $\widetilde{A}(0)=A$ and $\Delta(\alpha, \lambda(\alpha)) \widetilde{A}(\alpha) \equiv 0$ for all sufficiently small $\alpha$; we differentiate it with respect to $\alpha$ and obtain

$$
\left[\Delta_{\alpha}(\alpha, \lambda(\alpha))+\lambda^{\prime}(\alpha) \Delta_{\lambda}(\alpha, \lambda(\alpha))\right] A(\alpha)+\Delta(\alpha, \lambda(\alpha)) A^{\prime}(\alpha)=0
$$


In particular, we have

$$
\left[\Delta_{\alpha}\left(0, i \beta_{0}\right)+\lambda^{\prime}(0) \Delta_{\lambda}\left(0, i \beta_{0}\right)\right] A+\Delta\left(0, i \beta_{0}\right) A^{\prime}(0)=0 .
$$

This, together with the fact that $\bar{B}^{T} \Delta\left(0, i \beta_{0}\right)=0$, implies that

$$
\bar{B}^{T} \Delta_{\alpha}\left(0, i \beta_{0}\right) A+\lambda^{\prime}(0) \bar{B}^{T} \Delta_{\lambda}\left(0, i \beta_{0}\right) A=0 .
$$

For each $\phi \in \operatorname{Fix}(\Sigma, \operatorname{Ker} \mathcal{L}), \phi=z q+\overline{z q}$, where $z=\langle p, \phi\rangle$. Let

$$
g(z, \alpha, \beta):=\langle p, \widetilde{\mathcal{B}}(z q+\overline{z q}, \alpha, \beta)\rangle .
$$

Thus, we only need to consider the existence of nontrivial solutions to $g(z, \alpha, \beta)=0$. It follows from (2.7) that

$$
g_{z}(0,0,0)=0, \quad g_{\bar{z}}(0,0,0)=0 .
$$

It is easy to see that $g(z, \alpha, \beta)$ is $S^{1}$-equivariant. Using a similar argument to that in [6], we can find two functions $\Re, \Im: \mathbb{R}^{3} \rightarrow \mathbb{R}$ such that

$$
g(z, \alpha, \beta)=\Re\left(|z|^{2}, \alpha, \beta\right) z+\Im\left(|z|^{2}, \alpha, \beta\right) i z .
$$

It follows from $g_{z}(0,0,0)=0$ that $\Re(0,0,0)=0$ and $\Im(0,0,0)=0$. Let $z=$ $r e^{i \theta}$. Then solving $g$ is equivalent to either solving $r=0$ or $\Re\left(r^{2}, \alpha, \beta\right)=0$ and $\Im\left(r^{2}, \alpha, \beta\right)=0$. In view of the implicitly defined function $W(v, \alpha, \beta)$, which vanishes through first order in $v=z q+\overline{z q}$, we have

$$
F(v+W(v, \alpha, \beta), \alpha, \beta)=-(1+\beta) \frac{d}{d t} D(\alpha) v_{t, \beta}+L(\alpha) v_{t, \beta}+O\left(|z|^{2}\right) .
$$

Therefore,

$$
\begin{aligned}
& F_{\alpha}(v+W(v, 0,0), 0,0)=\Omega v_{t}+O\left(|z|^{2}\right), \\
& F_{\beta}(v+W(v, 0,0), 0,0)=\Xi v_{t}+O\left(|z|^{2}\right),
\end{aligned}
$$

where operators $\Omega=-\frac{d}{d t} D^{\prime}(0)+L^{\prime}(0), \Xi v_{t}=\frac{\partial}{\partial \beta}\left[-(1+\beta) \frac{d}{d t} D(\alpha) v_{t, \beta}+L(\alpha) v_{t, \beta}\right]_{\beta=0}$, $D^{\prime}(0)=\left.\frac{d}{d \alpha} D(\alpha)\right|_{\alpha=0}$, and $L^{\prime}(0)=\left.\frac{d}{d \alpha} L(\alpha)\right|_{\alpha=0}$. Notice that

$$
\begin{aligned}
\langle p, \Omega q\rangle= & \left\langle B e^{i \beta_{0} t}, i \beta_{0} \int_{-\tau}^{0} d \mu_{\alpha}(0, \theta) A e^{i \beta_{0}(t+\theta)}+\int_{-\tau}^{0} d \eta_{\alpha}(0, \theta) A e^{i \beta_{0}(t+\theta)}\right\rangle \\
= & \bar{B}^{T} \int_{-\tau}^{0}\left[i \beta_{0} d \mu_{\alpha}(0, \theta)+d \eta_{\alpha}(0, \theta)\right] A e^{i \beta_{0} \theta} \\
= & -\bar{B}^{T} \Delta_{\alpha}\left(0, i \beta_{0}\right) A, \\
\langle p, \Xi q\rangle= & -\left\langle B e^{i \beta_{0} t}, i \beta_{0} e^{i \beta_{0} t}-i \beta_{0} \int_{-\tau}^{0} d \mu(0, \theta) A\left[1+i \beta_{0} \theta\right] e^{i \beta_{0}(t+\theta)}\right\rangle \\
& +i \beta_{0} \bar{B}^{T} \int_{-\tau}^{0} d \eta(0, \theta) A \theta e^{i \beta_{0} \theta} \\
= & -i \beta_{0} \bar{B}^{T} A+i \beta_{0} \bar{B}^{T} \int_{-\tau}^{0} d \mu(0, \theta) A\left[1+i \beta_{0} \theta\right] e^{i \beta_{0} \theta} \\
& +i \beta_{0} \bar{B}^{T} \int_{-\tau}^{0} d \eta(0, \theta) A \theta e^{i \beta_{0} \theta} \\
= & -i \beta_{0}(p, q)
\end{aligned}
$$

and $(p, q)=\bar{B}^{T} \Delta_{\lambda}\left(0, i \beta_{0}\right) A$. It follows from (2.8) that $\langle p, \Omega q\rangle=(p, q) \lambda^{\prime}(0)$. Similarly, we have $\langle p, \Omega \bar{q}\rangle=\langle p, \Xi \bar{q}\rangle=0$. Therefore,

$$
\begin{aligned}
& g_{\alpha}(z, 0,0)=\left\langle p, F_{\alpha}(v, 0,0)\right\rangle=z(p, q) \lambda^{\prime}(0)+O\left(|z|^{2}\right), \\
& g_{\beta}(z, 0,0)=\left\langle p, F_{\beta}(v, 0,0)\right\rangle=-i \beta_{0} z(p, q)+O\left(|z|^{2}\right) .
\end{aligned}
$$

Then

$$
\begin{array}{ll}
\Re_{\alpha}(0,0,0)=\operatorname{Re}\left\{\lambda^{\prime}(0)(p, q)\right\}, & \Im_{\alpha}(0,0,0)=\operatorname{Im}\left\{\lambda^{\prime}(0)(p, q)\right\}, \\
\Re_{\beta}(0,0,0)=\beta_{0} \operatorname{Im}\{(p, q)\}, & \Im_{\beta}(0,0,0)=-\beta_{0} \operatorname{Re}\{(p, q)\} .
\end{array}
$$


So the Jacobi determinant of the functions $\Re$ and $\Im$ with respect to $\alpha$ and $\beta$ is

$$
\operatorname{det}\left[\begin{array}{cc}
\Re_{\alpha}(0,0,0) & \Re_{\beta}(0,0,0) \\
\Im_{\alpha}(0,0,0) & \Im_{\beta}(0,0,0)
\end{array}\right]=-\beta_{0} \operatorname{Re}\left\{\lambda^{\prime}(0)\right\}|(p, q)|^{2} .
$$

Thus, under condition (H4), the above Jacobi determinant is nonzero. The implicit function theorem implies that there exists a unique function $\alpha=\alpha\left(r^{2}\right)$ and $\beta=$ $\beta\left(r^{2}\right)$ satisfying $\alpha(0)=0$ and $\beta(0)=0$ such that

$$
\Re\left(r^{2}, \alpha\left(r^{2}\right), \beta\left(r^{2}\right)\right) \equiv 0, \quad \Im\left(r^{2}, \alpha\left(r^{2}\right), \beta\left(r^{2}\right)\right) \equiv 0
$$

for all sufficiently small $r$. Therefore, $g\left(z, \alpha\left(|z|^{2}\right), \beta\left(|z|^{2}\right)\right) \equiv 0$ for $z$ sufficiently near 0 . Therefore, system (1.1) has a bifurcation of periodic solutions whose spatiotemporal symmetry can be completely characterized by $\Sigma$. This completes the proof of Theorem 2.3 .

Remark 2.4. Theorem 2.3 implies that a Hopf bifurcation for (1.1) occurs at $\alpha=0$. Namely, in every neighborhood of $(x=0, \alpha=0)$ there is a branch of $\Sigma$-symmetric periodic solutions $x(t, \alpha)$ with $x(t, \alpha) \rightarrow 0$ as $\alpha \rightarrow 0$. The period $\omega_{\alpha}$ of $x(t, \alpha)$ satisfies that $\omega_{\alpha} \rightarrow \omega_{0}$ as $\alpha \rightarrow 0$. Moreover, $\Gamma$-equivariance implies that there are $\left(\Gamma \times S^{1}\right) / \Sigma$ different periodic solutions, which have isotopy subgroups conjugate to $\Sigma$ in $\Gamma \times S^{1}$.

In what follows, we consider the bifurcation direction. Assuming sufficient smoothness of $h$ and $f$, we write

$$
\begin{aligned}
h(0, \varphi) & =D(0) \varphi+\frac{1}{2} \mathcal{B}(\varphi, \varphi)+\frac{1}{6} \mathcal{C}(\varphi, \varphi, \varphi)+o\left(\|\varphi\|^{3}\right), \\
f(0, \varphi) & =L(0) \varphi+\frac{1}{2} \mathcal{E}(\varphi, \varphi)+\frac{1}{6} \mathcal{F}(\varphi, \varphi, \varphi)+o\left(\|\varphi\|^{3}\right) .
\end{aligned}
$$

In view of (2.5), we have $P F(z q+\overline{z q}+W(z q+\overline{z q}, \alpha, \beta), \alpha, \beta) \equiv 0$. Write $W(z q+$ $\overline{z q}, 0,0)$ and $g(z, 0,0)$ as

$$
W(z q+\overline{z q}, 0,0)=\sum_{s+l \geq 2} \frac{1}{s ! l !} W_{s l} z^{s} \bar{z}^{l}, \quad g(z, 0,0)=\sum_{s+l \geq 2} \frac{1}{s ! l !} g_{s l} z^{s} \bar{z}^{l} .
$$

It follows from (2.10) that $g_{21}=\Re_{1}(0,0,0)+i \Im_{1}(0,0,0)$, where $\Re_{1}(u, \alpha, \beta)=$ $\Re_{u}(u, \alpha, \beta)$ and $\Im_{1}(u, \alpha, \beta)=\Im_{u}(u, \alpha, \beta)$. Therefore, $\Re_{1}(0,0,0)=\operatorname{Re}\left\{g_{21}\right\}$ and $\Im_{1}(0,0,0)=\operatorname{Im}\left\{g_{21}\right\}$. From (2.11), we can calculate the derivatives of $\alpha\left(r^{2}\right)$ and $\beta\left(r^{2}\right)$ and evaluate at $r=0$ :

$$
\alpha^{\prime}(0)=-\frac{\operatorname{Re}\left\{\overline{(p, q)} g_{21}\right\}}{\operatorname{Re}\left\{\lambda^{\prime}(0)\right\}|(p, q)|^{2}}, \quad \beta^{\prime}(0)=-\frac{\operatorname{Im}\left\{\lambda^{\prime}(0)(p, q) \overline{g_{21}}\right\}}{\operatorname{Re}\left\{\lambda^{\prime}(0)\right\}|(p, q)|^{2}} .
$$

The bifurcation direction is determined by $\operatorname{sign} \alpha^{\prime}(0)$, and the monotonicity of the period of the bifurcating closed invariant curve depends on $\operatorname{sign} \beta^{\prime}(0)$. Using a similar argument to that in [5], we have

$$
\begin{aligned}
g_{21}= & \left\langle p, \mathcal{F}(q, q, \bar{q})-\frac{d}{d t} \mathcal{C}(q, q, \bar{q})\right\rangle \\
& +2\left\langle p, \mathcal{E}\left(q, W_{11}\right)-\frac{d}{d t} \mathcal{B}\left(q, W_{11}\right)\right\rangle \\
& +\left\langle p, \mathcal{E}\left(\bar{q}, W_{20}\right)-\frac{d}{d t} \mathcal{B}\left(\bar{q}, W_{20}\right)\right\rangle .
\end{aligned}
$$

We still need to compute $W_{11}$ and $W_{20}$. In fact, it follows that

$$
\begin{aligned}
& W_{20}=-\mathcal{L}^{-1} P\left\{-\frac{d}{d f} \mathcal{B}(q, q)+\mathcal{E}(q, q)\right\}, \\
& W_{11}=-\mathcal{L}^{-1} P\left\{-\frac{d}{d t} \mathcal{B}(q, \bar{q})+\mathcal{E}(q, \bar{q})\right\} .
\end{aligned}
$$


In order to evaluate function $W_{20}$, we must solve the following differential equations:

$$
\frac{d}{d t} D(0) W_{20}-L(0) W_{20}=P\left\{-\frac{d}{d t} \mathcal{B}(q, q)+\mathcal{E}(q, q)\right\} .
$$

Note that $\mathcal{B}(q, q)=\mathcal{B}\left(A e^{i \beta_{0}(\cdot)}, A e^{i \beta_{0}(\cdot)}\right) e^{2 i \beta_{0} t}$ and that $\mathcal{E}(q, q)=\mathcal{E}\left(A e^{i \beta_{0}(\cdot)}\right.$, $\left.A e^{i \beta_{0}(\cdot)}\right) e^{2 i \beta_{0} t}$. So, $g_{20}=\left\langle p,-\frac{d}{d t} \mathcal{B}(q, q)+\mathcal{E}(q, q)\right\rangle=0$. Namely, $-\frac{d}{d t} \mathcal{B}(q, q)+$ $\mathcal{E}(q, q) \in$ Range $\mathcal{L}$. Hence, the projection $P$ on $-\frac{d}{d t} \mathcal{B}(q, q)+\mathcal{E}(q, q)$ acts as the identity, and (2.12) is an inhomogeneous difference equation with constant coefficients. Thus, there is a particular solution of (2.12) of the form $W_{20}^{*}(t)=D_{2} e^{2 i \beta_{0} t}$. Substituting $W_{20}^{*}$ into (2.12) and comparing the coefficients, we obtain

$$
D_{2}=\Delta^{-1}\left(0,2 i \beta_{0}\right)\left\{\mathcal{E}\left(A e^{i \beta_{0}(\cdot)}, A e^{i \beta_{0}(\cdot)}\right)-2 i \beta_{0} \mathcal{B}\left(A e^{i \beta_{0}(\cdot)}, A e^{i \beta_{0}(\cdot)}\right)\right\} .
$$

In addition, $W_{20}^{*}$ is orthogonal to $p$, so it belongs to Range $\mathcal{L}$. Thus $W_{20}(0,0,0)$ is equal to $W_{20}^{*}$ with $D_{2}$ determined by (2.13). Similarly, we have

$$
g_{02}=g_{11}=0, \quad W_{02}=\bar{D}_{2} e^{-2 i \beta_{0} t}, \quad W_{11}=D_{0},
$$

where $D_{0}=\Delta^{-1}(0,0) \mathcal{E}\left(A e^{i \beta_{0}(\cdot)}, \bar{A} e^{-i \beta_{0}(\cdot)}\right)$. Therefore,

$$
\begin{aligned}
g_{21}= & \bar{B}^{T} \mathcal{F}\left(A e^{i \beta_{0}(\cdot)}, A e^{i \beta_{0}(\cdot)}, \bar{A} e^{-i \beta_{0}(\cdot)}\right)-i \beta_{0} \bar{B}^{T} \mathcal{C}\left(A e^{i \beta_{0}(\cdot)}, A e^{i \beta_{0}(\cdot)}, \bar{A} e^{-i \beta_{0}(\cdot)}\right) \\
& +2 \bar{B}^{T} \mathcal{E}\left(A e^{i \beta_{0}(\cdot)}, D_{0}\right)-2 i \beta_{0} \bar{B}^{T} \mathcal{B}\left(A e^{i \beta_{0}(\cdot)}, D_{0}\right) \\
& +\bar{B}^{T} \mathcal{E}\left(\bar{A} e^{-i \beta_{0}(\cdot)}, D_{2}\right)-i \beta_{0} \bar{B}^{T} \mathcal{B}\left(\bar{A} e^{-i \beta_{0}(\cdot)}, D_{2}\right) .
\end{aligned}
$$

We summarize the above discussion as follows.

Theorem 2.5. In addition to conditions $(\mathrm{H} 1)-(\mathrm{H} 4)$, assume that $L(\alpha)$ and $f(\alpha, \cdot)$ are sufficiently smooth. Then there exists a branch of $\Sigma$-symmetric periodic solutions, parameterized by $\alpha$, bifurcating from the trivial solution $x=0$ of (1.1). Moreover,

(i) $\operatorname{Re}\left\{\lambda^{\prime}(0)\right\} \operatorname{Re}\left\{\overline{(p, q)} g_{21}\right\}$ determines the direction of the bifurcation: the bifurcation is supercritical (respectively, subcritical); i.e., the bifurcating periodic solutions exist for $\alpha>0$ (respectively, <0) if $\left.\operatorname{Re}\left\{\lambda^{\prime}(0)\right\}\right\} \operatorname{Re}\left\{\overline{(p, q)} g_{21}\right\}$ $<0$ (respectively, $>0)$.

(ii) $\operatorname{Re}\left\{\lambda^{\prime}(0)\right\} \operatorname{Im}\left\{\lambda^{\prime}(0)(p, q) \overline{g_{21}}\right\}$ determines the period of the bifurcating periodic solutions along the branch: the period is greater than (respectively, less than) $\omega_{0}$ if it is positive (respectively, negative).

Example 2.6 (Application of Theorems 2.3 and 2.5 to ODEs). Consider the following system:

$$
\left[\begin{array}{c}
\dot{x} \\
\dot{y}
\end{array}\right]=\left[\begin{array}{cc}
\alpha & \beta_{0} \\
-\beta_{0} & \alpha
\end{array}\right]\left[\begin{array}{l}
x \\
y
\end{array}\right]+\left[\begin{array}{l}
f^{1}(x, y) \\
f^{2}(x, y)
\end{array}\right]
$$

where $\beta_{0}>0$, and $f^{j}$ is three times differentiable and satisfies $f_{x}^{j}(0,0)=f_{y}^{j}(0,0)=$ $0, j=1,2$. It is easy to see that if $\alpha=0$, then at the origin $(0,0)$, the linearized system of (2.14) has a solution basis $\left\{e_{1}, e_{2}\right\}$, where $e_{1}(t)=\left(\cos \left(\beta_{0} t\right),-\sin \left(\beta_{0} t\right)\right)^{T}$ and $e_{2}(t)=\left(\sin \left(\beta_{0} t\right), \cos \left(\beta_{0} t\right)\right)^{T}$. If we choose $q=2 p=(1, i)^{T} e^{i \beta_{0}(\cdot)}$ and denote $\operatorname{Re}\left\{g_{21}\right\}$ by $\Upsilon$, then $(p, q)=1$ and

$$
\begin{aligned}
\Upsilon= & f_{x x x}^{1}+f_{x y y}^{1}+f_{x x y}^{2}+f_{y y y}^{2} \\
& +\frac{1}{\beta_{0}}\left[f_{x y}^{1}\left(f_{x x}^{1}+f_{y y}^{1}\right)-f_{x y}^{2}\left(f_{x x}^{2}+f_{y y}^{2}\right)-f_{x x}^{1} f_{x x}^{2}+f_{y y}^{1} f_{y y}^{2}\right] .
\end{aligned}
$$


It follows from Theorem 2.5 that there exists a branch of periodic solutions of (2.14) bifurcating from the trivial solution $x=0$, and that $\Upsilon$ determines the directions of the Hopf bifurcation: the Hopf bifurcation is supercritical (subcritical); i.e., the bifurcating periodic solutions exist for $\alpha>0$ (respectively, $<0$ ) if $\Upsilon<0$ (respectively, $>0$ ). It should be noticed that the above $\Upsilon$ is exactly the one derived by Hassard and Wan [10] by using center manifold reduction and normal form approach (for more details, see [7).

\section{Application to a Ring Network}

To illustrate the results presented above, we consider a ring network consisting of $n$ identical elements with time-delayed nearest-neighbour coupling [12]:

$$
\left[u_{j}(t)-c u_{j}(t-1)\right]^{\prime}=g\left(u_{j+1}(t-1)\right)+g\left(u_{j-1}(t-1)\right)-3 g\left(u_{j}(t-1)\right),
$$

where $i(\bmod n), g \in C^{3}(\mathbb{R} ; \mathbb{R})$ with $g(0)=g^{\prime \prime}(0)=0$ and $g^{\prime}(0)=b>0$, and $c \in[0,1)$ is the bifurcation parameter. Define the action of the dihedral group $\mathbb{D}_{n}$ on $\mathbb{R}^{n}$ by

$$
(\rho \cdot u)_{j}=u_{j+1} \quad \text { and } \quad(\kappa \cdot u)_{j}=u_{2-j}
$$

for all $j(\bmod n)$ and $u \in \mathbb{R}^{N}$. It is easy to see that system (3.1) is $\mathbb{D}_{n}$-equivariant (see [8, 12]). Let $\mathcal{A}(c)$ be the infinitesimal generator of the linear operator generated by the linearization of (3.1) about the trivial solution $u=0$. It can be shown [12] that $\lambda \in \mathbb{C}$ is an eigenvalue of $\mathcal{A}(c)$ if and only if $\prod_{j=0}^{n-1} p_{j}(\lambda, c)=0$, where $p_{j}(\lambda, c)=\lambda+\left(\vartheta_{j}-c \lambda\right) e^{-\lambda}$ and $\vartheta_{j}=b+4 b \sin ^{2}(2 j \pi / n)>0$.

For a given $j, p_{j}(\cdot, c)$ has a pair of purely imaginary zeros $\pm \beta_{0}$ if $c=\cos \beta_{0}$ and $\vartheta_{j}=\beta_{0} \sin \beta_{0}$. This results in a family of bifurcation values $c_{j, k}$ in the interval $[0,1)$, where $c_{j, k}=\cos \beta_{j, k}$ for $k \in \mathbb{N}$, and $\left\{\beta_{j, k}\right\}_{k=1}^{\infty}$ is a strictly increasing sequence of positive numbers satisfying $\vartheta_{j}=\beta_{j, k} \sin \beta_{j, k}$ for all $k \in \mathbb{N}$ and $\lim _{k \rightarrow \infty} \beta_{j, k}=\infty$. Moreover, if $\lambda(c)$ is a smooth curve of zeros of $p_{j}(\cdot, c)$ with $\lambda\left(c_{j, k}\right)=i \beta_{j, k}$, it is easy to see that

$$
\lambda^{\prime}\left(c_{j, k}\right)=D\left(c_{j, k} \beta_{j, k}^{2}-i \vartheta_{j} \beta_{j, k}\right),
$$

where $D=\left|\left(\cos \beta_{j, k}+i \sin \beta_{j, k}\right)\left(1+\beta_{j, k}\right)-c_{j, k}\right|^{-2}$. Therefore, for fixed $j$ and $k, \mathcal{A}\left(c_{j, k}\right)$ has a pair of purely imaginary eigenvalues $\pm i \beta_{j, k}$ with the associated eigenspace $E_{0}$ spanned by the eigenvectors $e^{i \beta_{j, k}(\cdot)} v_{j}, e^{i \beta_{j, k}(\cdot)} \overline{v_{j}}, e^{-i \beta_{j, k}(\cdot)} v_{j}$ and $e^{-i \beta_{j, k}(\cdot)} \overline{v_{j}}$, where $v_{j}=\left(1, e^{2 \pi i / n}, \ldots, e^{2(n-1) j \pi / n}\right)$. Thus, assumptions (H1), (H2), and (H4) hold. If $j \neq 0$ and $j \neq n / 2$, it furthermore follows from 8 ] that

$$
\begin{aligned}
& \operatorname{Fix}\left(\Sigma_{\kappa}^{+}\right)=\operatorname{span}\left\{w_{1} \cos \left(\beta_{j, k} t\right), w_{1} \sin \left(\beta_{j, k} t\right)\right\}, \\
& \operatorname{Fix}\left(\Sigma_{\kappa}^{-}\right)=\operatorname{span}\left\{w_{2} \cos \left(\beta_{j, k} t\right), w_{2} \sin \left(\beta_{j, k} t\right)\right\}, \\
& \operatorname{Fix}\left(\Sigma_{\rho}^{+}\right)=\operatorname{span}\left\{\operatorname{Re}\left(\overline{v_{j}} e^{i \beta_{j, k} t}\right), \operatorname{Im}\left(\overline{v_{j}} e^{i \beta_{j, k} t}\right)\right\}, \\
& \operatorname{Fix}\left(\Sigma_{\rho}^{-}\right)=\operatorname{span}\left\{\operatorname{Re}\left(v_{j} e^{i \beta_{j, k} t}\right), \operatorname{Im}\left(v_{j} e^{i \beta_{j, k} t}\right)\right\},
\end{aligned}
$$

where $w_{1}=\operatorname{Re}\left(v_{j}\right), w_{2}=\operatorname{Im}\left(v_{j}\right)$, and $\Sigma_{\kappa}^{ \pm}=(\kappa, \pm 1)$ and $\Sigma_{\rho}^{ \pm}=\left(\rho, e^{ \pm 2 i j \pi /\left(n \beta_{j, k}\right)}\right)$ are subgroups of $\mathbb{D}_{n} \times S^{1}$. Thus, all conditions of the equivariant Hopf bifurcation theorem (Theorem 2.3) are satisfied. Therefore, we apply Theorem 2.3 to system (3.1) and obtain the following results. 
Theorem 3.1. (i) Near $c=c_{0, k}$ for each $k \in \mathbb{N}$, there exists a branch of synchronous periodic solutions of period $\omega$ near $\left(2 \pi / \beta_{0, k}\right)$ bifurcated from the zero solution of the system. (ii) Near $c=c_{j, k}$ for each $j \in\{1,2, \ldots,[(n-1) / 2]\}$ and $k \in \mathbb{N}$, there exist $2(n+1)$ branches of asynchronous periodic solutions of period $\omega$ near $\left(2 \pi / \beta_{j, k}\right)$ bifurcated from the zero solution of the system, and these are two phaselocked waves, $n$ mirror-reflecting waves, and $n$ standing waves.

In what follows, we start with the two phase-locked oscillations mentioned above, which are characterized by $\Sigma_{\rho}^{ \pm}$. In view of (3.4), for the vectors $A$ and $B$ defined in Section 2, we choose $A=n B=\bar{v}_{j}$ or $A=n B=v_{j}$. We have

$$
\operatorname{Re}\left\{\overline{(p, q)} g_{21}\right\}=\vartheta_{j}^{2} g^{\prime \prime \prime}(0) \text { and } \operatorname{Im}\left\{\lambda^{\prime}(0)(p, q) \overline{g_{21}}\right\}=-g^{\prime \prime \prime}(0) \vartheta_{j} \beta_{j, k} .
$$

Similarly, we choose $A=\frac{n}{2} B=w_{1}$ for the mirror-reflecting waves characterized by $\Sigma_{\kappa}^{+}$, and $A=\frac{n}{2} B=w_{2}$ for the mirror-reflecting waves characterized by $\Sigma_{\kappa}^{-}$. By a direct computation, we have

$$
\operatorname{Re}\left\{\overline{(p, q)} g_{21}\right\}=\frac{m}{4} \vartheta_{j}^{2} g^{\prime \prime \prime}(0) \text { and } \operatorname{Im}\left\{\lambda^{\prime}(0)(p, q) \overline{g_{21}}\right\}=-\frac{m}{4} g^{\prime \prime \prime}(0) \vartheta_{j} \beta_{j, k},
$$

where $m=4$ if $4 j=0(\bmod n)$, or $m=3$ otherwise.

Finally, for the synchronous periodic solution mentioned in Theorem 3.1, we can show that (3.5) holds for $j=0$. Thus, applying Theorem 2.5, we have the following results.

Theorem 3.2. Near $c=c_{j, k}$ for each $j \in\{0,1, \ldots, n-1\}$ and $k \in \mathbb{N}$, system (3.1) undergoes a Hopf bifurcation; both the bifurcation direction and the period of bifurcating periodic solutions are determined by the sign of $g^{\prime \prime \prime}(0)$. More precisely, if $g^{\prime \prime \prime}(0)<0$ (or $>0$ ), then (i) the Hopf bifurcation is supercritical (respectively, subcritical) and all the bifurcating periodic solutions exist for $c>c_{j, k}$ (respectively, $\left.<c_{j, k}\right)$; (ii) the period of each branch of bifurcating periodic solutions is greater (respectively, less) than $\left(2 \pi / \beta_{j, k}\right)$.

\section{ACKNOWLEDGMENTS}

Part of this work was done while the first author was a postdoctoral fellow at Imperial College London, supported by the UK Royal Society. He is grateful for the hospitality of the Department of Mathematics of Imperial College London during this period.

\section{REFERENCES}

1. N. Chafee, A bifurcation problem for a functional differential equation of finitely retarded type, J. Math. Anal. Appl., 35 (1971), 312-348. MR0277854 (43:3587)

2. S. N. Chow and J. Mallet-Paret, Integral averaging and bifurcation, J. Diff. Eqn., 26 (1977), 112-159. MR0488151 (58:7718)

3. T. Faria and L. T. Magalhães, Normal forms for retarded functional-differential equations with parameters and applications to Hopf bifurcation, J. Diff Eqn., 122 (1995), 181-200. MR.1355888 (97a:34186b)

4. K. Geba, W. Krawcewicz, and J. Wu, An equivariant degree with applications to symmetric bifurcation problems, I: Construction of the degree, Proc. London Math. Soc., 69 (1994), 377-398. MR 1281970 (95g:58025)

5. M. Golubitsky and D. G. Schaeffer, Singularities and Groups in Bifurcation Theory, Vol. 1, Springer-Verlag, New York, 1985. MR771477 (86e:58014)

6. M. Golubitsky, I. Stewart and D. G. Schaeffer, Singularities and Groups in Bifurcation Theory, Vol. 2, Springer-Verlag, New York, 1988. MR950168 (89m:58038) 
7. J. Guckenheimer and P. J. Holmes, Nonlinear Oscillations: Dynamical System and Bifurcations of Vector Fields, Springer-Verlag, New York, 1983. MR709768 (85f:58002)

8. S. Guo and L. Huang, Hopf bifurcating periodic orbits in a ring of neurons with delays, Physica D., 183 (2003), 19-44. MR2006470 (2004i:37168)

9. J. Hale, Theory of Functional Differential Equations, Springer-Verlag, New York, 1977. MR0508721 (58:22904)

10. B. D. Hassard and Y. Wan, Bifurcation formulae derived from center manifold theory, J. Math. Anal. Appl., 63 (1978), 297-312. MR0488152 (58:7719)

11. E. Hopf, Abzweigung einer periodischen Lösung von einer stationären eines Differentialsystems, Berichen Math. Phys. Kl. Säch. Akad. Wiss. Leipzig, 95 (1943), 3-22. MR0039141 $(12: 501 \mathrm{c})$

12. W. Krawcewicz, S. Ma and J. Wu, Multiple slowly oscillating periodic solutions in coupled lossless transmission lines. Nonlinear Anal. Real World Appl., 5 (2004), no. 2, 309-354. MR2025070 (2004i:34183)

13. M. Weedermann, Hopf bifurcation calculations for scalar neutral delay differential equations, Nonlinearity, 19 (2006), 2091-2102. MR2256652 (2007g:34149)

14. J. Wu, Symmetric functional differential equations and neural networks with memory, Trans. Amer. Math. Soc., 350 (1998), 4799-4838. MR1451617(99i:34104)

College of Mathematics and Econometrics, Hunan University, Changsha, Hunan 410082, People's Republic of China

E-mail address: shangjguo@hnu.cn

Department of Mathematics, Imperial College London, London SW7 2AZ, United KINGDOM

E-mail address: jeroen.lamb@imperial.ac.uk 